\title{
GC Calibration and Instrumentation Involved.
}

\author{
Syed Shoeb ur Rahman ${ }^{\# 1}$, Dr. C Gurudas Nayak ${ }^{* 2}$, Yuvuraju Kuruba ${ }^{\# 3}$ \\ ${ }^{\# 1}$ Department of Instrumentation and Control, ${ }^{\# 2}$ Department of Instrumentation and Control, \\ \#3 Department of Analytical Instrumentation \\ ${ }^{1}$ syed.shoeb1@learner.manipal.edu \\ 2.cg.nayak@manipal.edu \\ 3 yuvuraju.kuruba@gvkbio
}

\begin{abstract}
In today's world chromatography is the foundation of separation of components used for further analysis. In chromatography we have different instruments being used which further depends upon the mobile phase which is usually either gas or liquid. The paper targets at the usage of gas chromatography over liquid chromatography under specified conditions. We are also using a calibration component whose mass is known to us. This paper also gives a lucid approach as to why gas chromatography is used.
\end{abstract}

Keyword- GC; LC

\section{INTRODUCTION}

A process involving bifurcation of components from the given pure drug by the usage of a gaseous mobile phase. It also involves a sample which is vaporized and further being injected onto a head of the chromatographic column. The sample is further transferred via a column by the flow of inert, gaseous mobile phase. The column contains a liquid stationary phase which is adsorbed onto the surface of an inert solid. Major criteria for the compounds to be analyzed by G.C are Volatility and Thermo stability. Two major types available are Gas-solid chromatography and Gas-liquid chromatography.

It is a process of bifurcating extremely minute components into its multifarious particles and identification of those particles with the application of electronic detectors.

Firstly a sample is being injected into a stream of helium which is later passed through a long column which is present in a heated chamber. The column slows down big molecules while letting small molecules move faster so they reach the detector sooner.

The columns are usually filled with fine clay or glass beads coated with oily materials that can slow down molecules based on their chemical make-up.

To understand the application of gas chromatography over liquid chromatography there are few difference between them which we need to understand. U.V inactive compounds contain conjugation that is double bond which are also referred as chromophore molecules. For lesser range of wavelength we use gas chromatography that is less than 200nm. For L.C (Liquid Chromatography) it is 200 to $400 \mathrm{~nm}$. A diluent of DCM (Dichloromethane) of chemical formula $\mathrm{CH}_{2} \mathrm{CL}_{2}$ or ACN (Acetonitrile) of chemical formula $\mathrm{CH}_{3} \mathrm{CN}$ is used. In liquid chromatography the buffers used will be $\mathrm{H}_{2} \mathrm{O}$, ACN and the samples are UV active which could be further recovered. Whereas in the case of gas chromatography we have He (Helium), $\mathrm{Na}$ (Sodium), Hydrogen and the samples are UV inactive and volatile compounds which can never be recovered in future.

Gas Chromatography (GC) involves the vaporization of molecules. The molecules are introduced in a column (typically an inert gas like He or sometimes a typically non-reactive one like N2). The column is typically coated with a thin layer of polar stationary phase. The more polar compounds will be more attracted to the stationary phase and thus will elute at a different time. The resultant spectrum will depict peaks that measure the time when each molecule eluted and the area of the peaks will give a rough estimation of the amount of each molecule.

The practical requirements for a gas chromatography are as follows:

$>$ Carrier gas

$>$ Flow regulators and Flow meters

$>$ Injection devices

$>$ Columns

$>$ Temperature control devices

$>$ Detectors

$>$ Recorders \& Integrators 


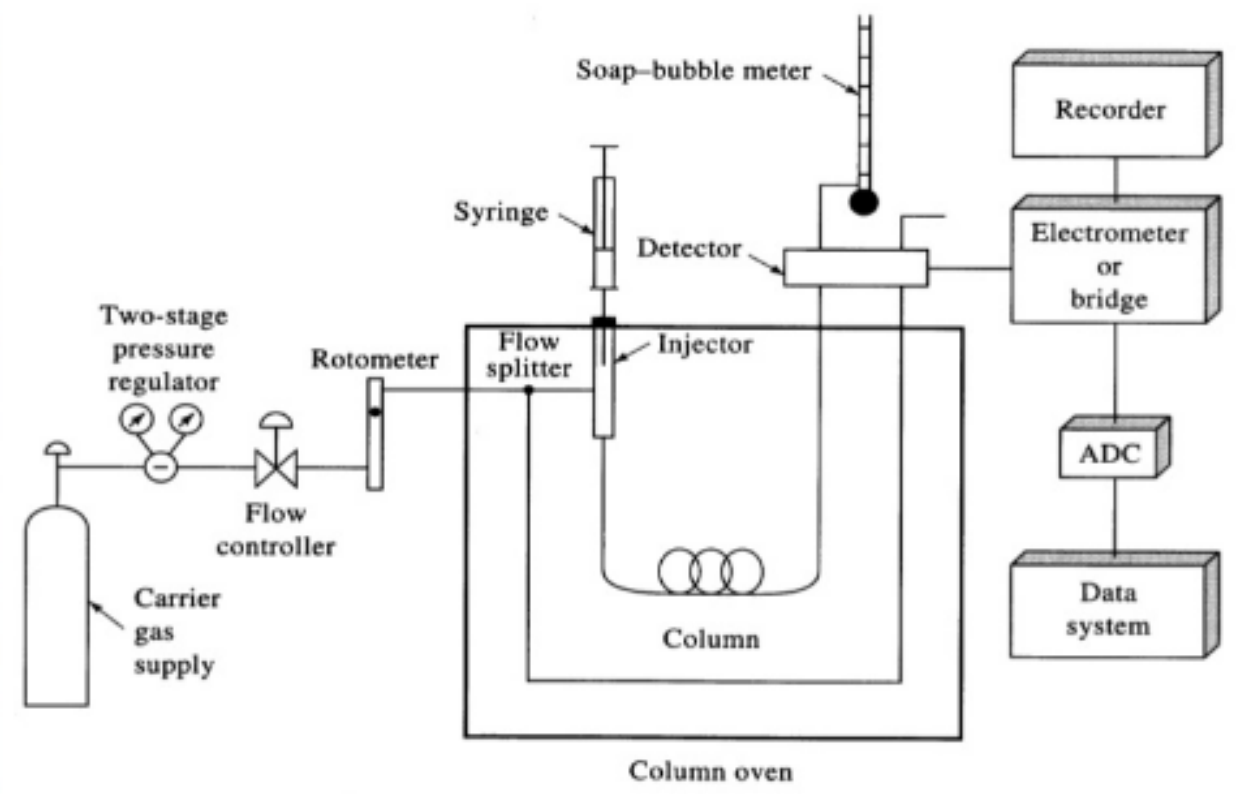

Fig. 1. Block diagram of Gas Chromatography

Gas chromatography s only used for solvent analysis. It is used for residual analysis and highly volatile compounds. In this process mobile phase is present inside the carrier gas tank. Now this is exposed to the sample injection inlet. This is further transmitted to the column stationary phase. The block diagram below explains it all.

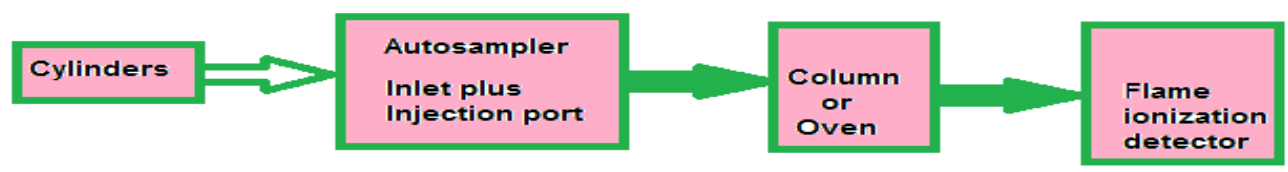

Fig. 2. Flow diagram of GC.

The instrument also consists of ALS (Automatic liquid Sampler) just after the gas regulator for the analysis of the liquid sample.

Hydrogen is used, as it has been already answered, hydrogen is used as combustible in the FID detector.

It is also used as a carrier gas in instruments equipped with a TCD (thermal conductibility) detector because it has the highest thermal conductivity of any gas.

However, it has two drawbacks: one, it is flammable, two, it may react with some molecules in the analyte thus skewing the results; for these reasons in the past helium was the preferred carrier because, while having the second-highest thermal conductivity, it is much safer than hydrogen and totally non-reactive.

Recently hydrogen is making a comeback due to the ever increasing cost - and decreasing availability - of helium.

The auto sampler consists of two components

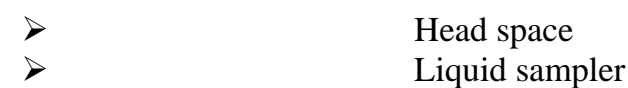

In head space the sample kept in same temperature for an hour, So volatile compounds gets evaporated and are further taken by the injector. There is no measurement taken. Whereas in the case of liquid sampler it directly takes it .At the inlet we have the septa and the liner. Septa function is to prevent gases from leakages as it might cause spikes in the chromatogram. These spikes are unwanted. The liner helps in sending the required amount of gas.

The sample after being processed will move to the splitter and later it enters the FID (Flame Ionization detector) or MS (Mass spectrometer). 


\section{PROPOSED WORK}

\section{Flame Ionization detector:}

\section{Fast Response FID}

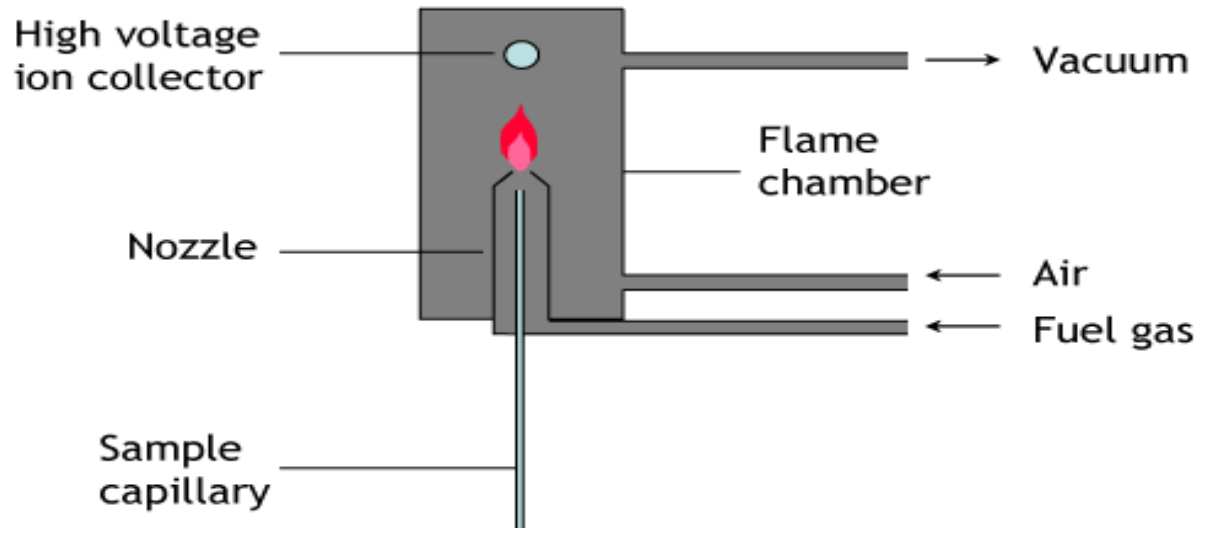

Fig. 3. Flame Ionization detector

This is one amongst the most frequently used detectors in gas chromatography. This used for flame production. Over here spark is introduced to the mixture of hydrogen and air for ignition process. It operates by the principle by the change in conductivity of the flame as the compound is burnt. The alteration in the conductivity of the flame does not arise by simple ionization of the compound. It is a partial or complete stripping of the compound to give charged hydrogen- deficient polymers or aggregates of carbon of low ionization potential.

In the flame produced samples are being sent for their ionization. Gases like nitrogen and helium are used as mobile phase. Because they are inert and do not react with samples. The fuel gas is hydrogen which generates flame. The flow is regulated by the flow regulators and later sample enters the flame and takes ions. Here volatile samples are used. Non-volatile samples are used in liquid chromatography. High voltage ion collection takes place and is sent to the $\mathrm{A} / \mathrm{D}$ converter for further processing.

The FID is a destructive technique that is the sample sent for testing will be intractable.

\section{EXPERIMENT AND RESULTS}

The heart of quantitative analysis is propercalibration. By determining the relationship between the magnitude of a peak for a known amount of analyte in a standard, one can then use that relationship (the calibration curve) to estimate the amount of that analyte in a sample of unknown concentration.

Calibration is basically done to check the accuracy of the system. Here the compounds used are:

16 Hexa decane

14 Tetra decane

Do decane

Components having less $\mathrm{CH}_{2}$ bonds are eluted. First we go for the tune page also called as the autotune, which shows the combustion techniques of FID. 


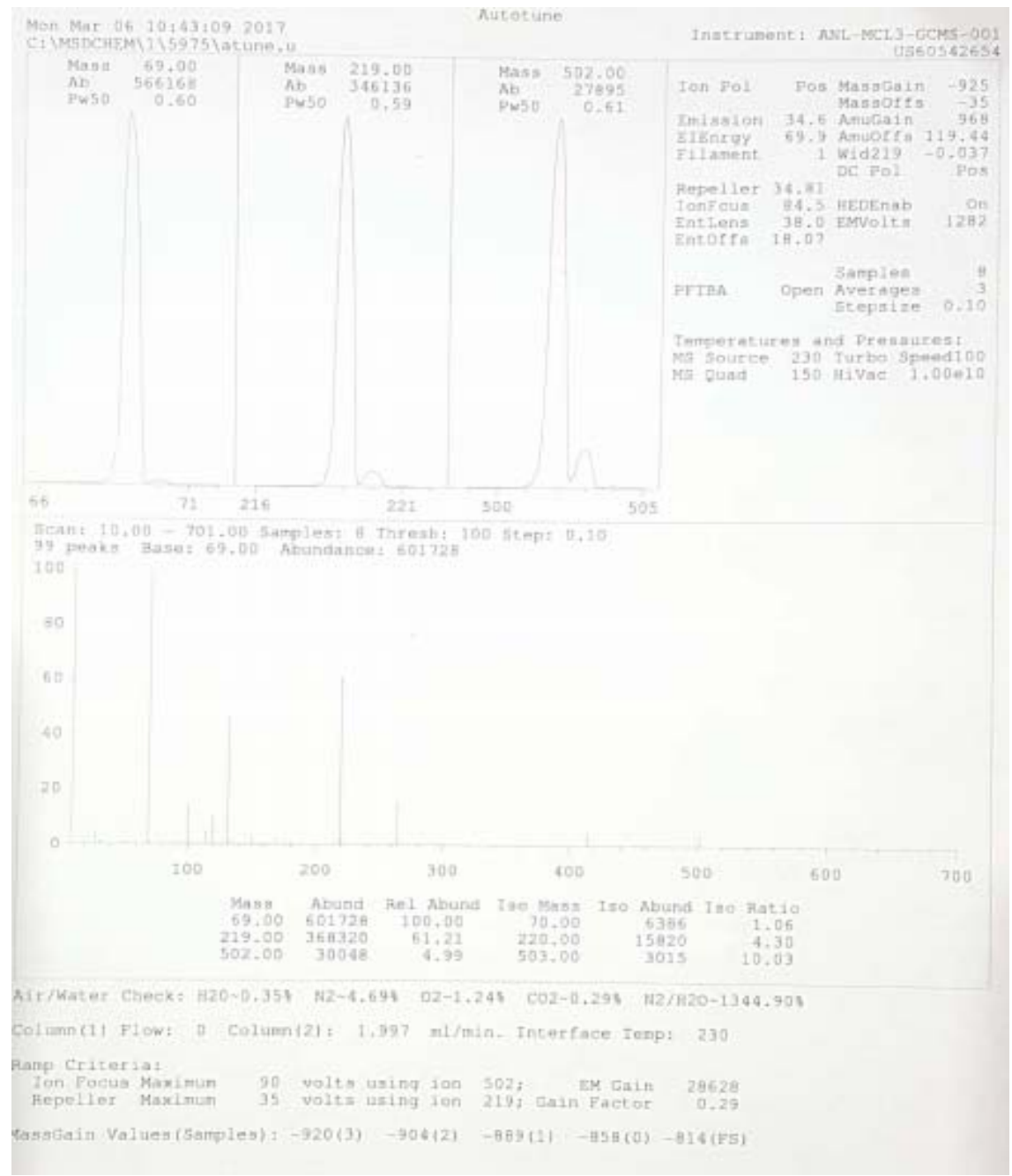

Fig. 4. Auto Tune page

Now we introduce samples to the gas chromatography instrument and obtain results for the calibration. In calibration we get the mass of those three compounds listed above. The $\mathrm{x}$-axis indicates the time whereas the $y$ axis indicates the response. The resultant is the slope or the retention time. For example over here 5.307 is the retention time.

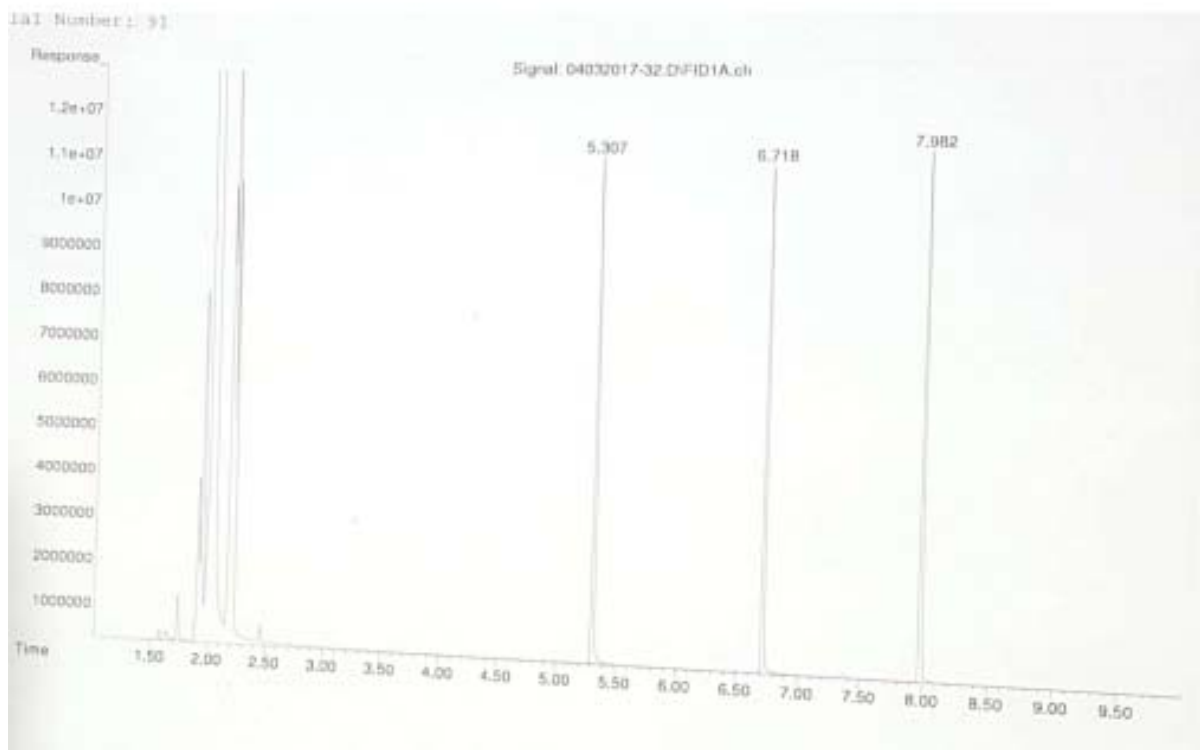

Fig. 5. Graphical analysis of a sample 


\section{CONCLUSION}

We have got a clear picture of how gas chromatography works. Therefore every instrument needs to be calibrated before we introduce a new sample for further tests. As we need to calibrate and validate the instrument before. The masses of the compounds are detected.

\section{ACKNOWLEDGMENT}

We sincerely thank the Department of Instrumentation and control Engineering at Manipal Institute of Technology, Manipal University, Manipal and GVK-BIOSCIENCE, Hyderabad for providing us with all the required inputs and the instruments to carry forward the case study. All the experimental analysis has been done at the GVK-BIOSCIENCE Labs.

\section{REFERENCES}

[1] Pati V.P, Tathe R.D, DEVDHE S.J (2011) “ULTRA PERFORMANCE LIQUID CHROMATOGRAPHY: A REVIEW.” International Research Journal Of Pharmacy. ISSN 2230 - 8407.

[2] Sharat Chandra, Selam, Priyanka.G, Nagarjun Reddy (2013) “Switch from HPLC to UPLC” International Journal of pharmaceutical science. $237-246$

[3] Kondawar M.S; Ultra Performance Liquid Chromatography: A faster and sensitive method over HPLC; Available from http://www.pharmainfo.net/

\section{AUTHOR PROFILE}

Author ${ }^{1}$ Syed Shoeb ur Rahman Student of Manipal University Author $^{2}$ Dr. C Gurudas Nayak Associate Professor Senior Scale - Manipal University. 